Sādhanā, Vol. 17, Part 1, March 1992, pp. 167-191. (C) Printed in India.

\title{
Levels of knowledge in distributed systems
}

\author{
ROHIT PARIKH ${ }^{1}$ and PAUL KRASUCKI ${ }^{2}$ \\ ${ }^{1}$ Department of Computer Science, CUNY Graduate Center, 33 West \\ 42nd Street, New York, NY 10036, USA \\ ${ }^{2}$ Department of Computer Science, Rutgers University, Camden College \\ of Arts and Sciences, Camden, NJ 08102, USA
}

\begin{abstract}
We correlate the level of knowledge of certain formulas in a group of individuals with certain regular, downward closed, sets of strings. We show that in suitable circumstances, all such sets can occur as levels of knowledge but that the lack of synchrony, or the lack of asynchrony when there are only two processors in the group, can create more or less severe restrictions.
\end{abstract}

Keywords. Levels of knowledge; distributed systems; common knowledge.

\section{Introduction}

It has been suggested recently that the notions of knowledge and common knowledge may be useful in analysing the behaviour of distributed systems and in designing protocols (Parikh \& Ramanujam 1985, pp. 256-268; Chandy \& Misra 1986; Parikh 1986, pp. 322-331; Halpern \& Zuck 1987, pp. 269-280; Moses \& Tuttle 1988; Halpern \& Moses 1990).

As specific examples, we cite the paper by Moses \& Tuttle (1988) who proved that certain synchronized action problems require common knowledge, and that there is always a most efficient solution which is an implementation of a simple knowledge based algorithm - an algorithm where there are explicit tests of knowledge. This algorithm is of the form "repeat... until $C_{U} A$ " for a certain formula $A .{ }^{1}$

Again, it was shown by Halpern \& Zuck (1987, pp. 269-280) that if a sequence of bits is communicated in an asynchronous system where messages can be delayed or lost (but if they are received, they are received in order), then to prove correctness of the protocol it is necessary and sufficient to prove that $K_{s} K_{r} K_{s} K_{r}$ ("value of the current bit") ${ }^{2}$ is true whenever the sender sends the next bit. Unlike the case with Moses \& Tuttle (1988), common knowledge is not necessary, nor would it be attainable if needed.

\footnotetext{
${ }^{1} C_{U} A$ means that there is common knowledge among the processes in $U$ that $A$ is true. The following important property of common knowledge is used in the synchronization algorithms: common knowledge is always achieved simultaneously by all the processes involved.

${ }^{2}$ The sender knows that the receiver knows that the sender knows that the receiver knows the value of the current bit.
} 
Other places where these notions have been found to be important are the semantics of natural language (Lewis 1969; Schiffer 1972) and mathematical economics (Aumann 1976; Parikh \& Krasucki 1990).

Since there are useful protocols which can be specified in terms of knowledge formulae, formulae of the form $K_{i_{1}} K_{i_{2}} \ldots K_{i_{m}} A$, where $K_{i_{j}}$ are knowledge operators, we investigate which sets of such formulae can occur as states (or levels) of knowledge for some formula $A$.

We define a logic of knowledge by augmenting some base logic $L$ (e.g. propositional logic) with modal operators $K_{i}$ for $i \leqslant n$. For every process $i, i$ knows $A$ if and only if according to $i$ 's view of the world, $A$ must be true.

We assume that processes' information is always correct, although often incomplete. Processes may not be able to describe precisely the global state of the system, some states may be indistinguishable to them, but they always admit the real state of the system as one of the possibilities.

This leads to a Kripke semantics where every process $i$ has some indistinguishability relation $R_{i}$ and in the state $s$, process $i$ knows that a formula $A$ is true iff for all the states $s^{\prime}$ which look the same to $i$ as $s, A$ is true in $s^{\prime}$. Since we deal with distributed systems, and we want to carry all the information about the past in our states, we will call the states histories. A history $H$ is a sequence of snapshots of the system, i.e. a sequence of $n$-tuples of actions ( $n$ is the number of processes), where a snapshot is taken at every tick of a very fine clock. If a process does nothing, its action is taken to be the special null action.

A first natural problem is the characterization of the sets of strings of knowledge operators $x$ such that for some fixed formula $A$ and history $H, H \vDash x A$.

This is a purely logical problem. The results are valid for every model of distributed systems where the indistinguishability relation $R_{i}$ for every process $i$ is an equivalence relation.

These sets of knowledge formulae (formulae of the form $K_{i} K_{j} \ldots . A$ ), we will call levels of knowledge.

The second problem is the design of a protocol, when possible, in which all the knowledge formulae (and exactly the knowledge formulae) in the set are satisfied.

To solve this problem we need to design a model of distributed system in which we can control the acquisition of knowledge. We need a system in which there is no accidental knowledge (or accidental synchrony), a system in which all the knowledge of the process about the others must be a result of some communications.

If processes in a distributed system know each other's programs, the lack of knowledge in the system may be due to: non-determinism, inputs, faults, communications and asynchrony.

In our system, lack of knowledge will be the result of three factors: initial private inputs of processes, lack of synchrony, possible delays in the communication system.

It turns out that the existence of a protocol depends on different assumed means of communication available in a distributed system.

We will analyse systems where all communications are asynchronous (there is uncertainty about the delivery time of a message); systems where all communications are instantaneous (synchronous systems); and systems where both types of communications are available.

The paper will consist of three main parts:

(1) Logic and levels of knowledge-description of the logic, definition of the level of knowledge of a formula, characterization of levels of knowledge. 
(2) Model of a distributed system - definition of the distributed system in which all knowledge about other processors is acquired through communication.

(3) Realization of levels of knowledge in distributed systems - given a set which may be the level of knowledge of some formula we design a protocol which realizes precisely that level.

Our work is a continuation and extension of the research initiated by Parikh (1986, pp. 322-331) and it includes the results from Parikh (1986, pp. 322-331) as well as Parikh \& Krasucki (1986).

\section{Logic and levels of knowledge}

A protocol is a set of global histories where global histories are finite sequences of events in the system, which are prefixes of runs of the system. $L_{0}$ is a language which describes properties of the global histories in protocol P. For every sentence $A$ in $L_{0}$, and for every history $H \in \mathbf{P}, A$ is either true or false in $H$.

We want to make sure that every processor's private information is expressible in our language. To accomplish that we assume that we have in our language a countable set of propositions $Q_{i, j}$, where $Q_{i, j}$ is the proposition that the $j$ th input value of processor $i$ is 1 . The $Q_{i, j}$ are independent but only finitely many can be true. The truth values of the $Q_{i, j}$ are the private facts of individual $i$. When we do not care about the 'owners' of these facts $Q_{i, j}$ then we shall refer to them just as the 'private facts' and denote them as $P_{k}$.

$L$ is the closure of $L_{0}$ under truth functional connectives. $L$ can be extended to a larger language $L_{K}$ which is the closure of $L$ under the knowledge operators $K_{i}$ (for $i \in N)$ and the usual truth functional connectives. Here $K_{i}(A)$ means that processor $i$ knows $A$.

The class of all models we consider is the class of all protocols $\mathbf{P}$ as described in the next section. Let's fix $P$. Now we define the notion $H \vDash A$ for $A$ in $L_{K}$ by recursion on the complexity of $A$. We assume that for every process there is some equivalence relation $\approx_{i}$ defined on histories. $H \approx_{i} H^{\prime}$ means that according to $i$ 's view $H$ and $H^{\prime}$ are indistinguishable.

(0) If $A$ is from $L_{0}$ then the semantics is given.

(1) If $A$ is $Q_{i, j}$ then $A$ is true in $H$ if the $j$ th bit of the input of processor $i$ in $H$ is 1 :

$$
H \vDash A, \quad \text { iff } H=\left(v_{1}, \ldots, v_{n}\right) ; H^{\prime},\left(v_{i}\right)_{j}=1 .
$$

(2) $A$ is $\neg A^{\prime}$ then $H \vDash A$, iff $H \# A^{\prime}$.

If $A$ is $B \vee C$ then $H \vDash A$, iff $(H \vDash B$ or $H \vDash C)$.

(3) $H \vDash K_{i} A$, iff $\forall H^{\prime} \in \mathbf{P} H \approx_{i} H^{\prime} \rightarrow H^{\prime} \vDash A$.

We will sometimes also need to refer to common knowledge operators $C_{U}$ where $U \subseteq N$. If $A$ is of the form $C_{U}(B)$, then $A$ is true in $H$ iff $B$ is true in every $H^{\prime}$ such that there is a chain of processors $i_{1}, \ldots, i_{k}$ in $U$ and a chain of histories $H=H_{1}, \ldots, H_{k}, H_{k+1}=$ $H^{\prime}$, such that for every $1 \leqslant j \leqslant k, H_{j} \approx_{i_{j}} H_{j+1}$.

We also define a new relation $\approx_{v}$ as follows:

$$
H \approx_{U} H^{\prime} \text { iff } \exists i \in U H \approx_{i} H^{\prime}
$$

Then $\approx_{U}^{*}$ will be the reflexive, transitive closure of $\approx_{U}$, and we have the following 
equivalent semantics for the common knowledge operator:

$$
H \vDash C_{U} A, \quad \text { iff } \forall H^{\prime} \in \mathbf{P}, \quad H \approx_{U}^{*} H^{\prime} \rightarrow H^{\prime} \vDash A .
$$

Note that if $U$ is empty, then $H \vDash C_{U} A$ iff $H \vDash A$, since $\approx_{U}^{*}$ is then the identity relation. For a set $X$ of formulas, we will write $H \vDash X$ to mean that for all $A \in X, H \vDash A$.

Since the $\approx_{i}$ are equivalence relations, the following S5 axioms and rules hold.

(1) All tautologies.

(2) $K_{i}(A) \wedge K_{i}(A \rightarrow B) \rightarrow K_{i}(B)$.

(3) $K_{i}(A) \rightarrow A$.

(4) $K_{i}(A) \rightarrow K_{i}\left(K_{i}(A)\right)$.

(5) $\neg K_{i}(A) \rightarrow K_{i}\left(\neg K_{i}(A)\right)$.

The rules modus ponens and necessitation are sound where the latter allows us to infer a formula $K_{i}(A)$ provided that we have shown that the formula $A$ holds for all histories $H$. This logic in the case when $L_{0}$ is propositional is known as $L K 5$.

The same axiom system will also be sound if we replace $K_{i}$ by $C_{U}$. In this case we can add one more axiom:

(6) $V \subseteq U \rightarrow\left(C_{U} A \rightarrow C_{V} A\right)$

If $U=\{i\}$ then $\approx_{i}$ and $\approx_{U}^{*}$ coincide since $\approx_{i}$ is transitive.

In the following characterization of levels of knowledge we will not need all the axioms: axiom 5 will not be used, so our results are valid also for the system in which $\approx_{i}$ are reflexive and transitive but not necessarily symmetric (the logic satisfies S4 axioms).

\subsection{Levels of knowledge}

In this section we show how to define the level of knowledge of a formula as a set of strings over the alphabet $\Sigma$ where $\Sigma=\left\{K_{1}, \ldots, K_{n}\right\}$ and investigate properties of levels of knowledge.

Consider a formula $A$ and some global history $H$. The formula may be true but not known to anyone. In that case we have $H \vDash A$ but not $H \vDash K_{i}(A)$ for any $i$. Or perhaps it may be known to some $i$ that $A$ is true. In the latter case, $K_{i}(A)$ is true and $A$ will hold at all histories $H^{\prime}$ such that $H^{\prime} \approx_{i} H$. The formula $K_{j} K_{i}(A)$ expresses the stronger assertion that not only is $A$ known to $i$, but also that this fact is known to $j$. Still more is known in the system if among $i$ and $j$ both know that both know,..., that both know $A$. This is common (or mutual) knowledge of $A$ between $i$ and $j$ and is denoted by $C_{\{i, j\}}(A)$. Thus a formula that is known may be known at a higher (in some sense) or lower level.

The highest possible level of knowledge here is $C_{\mathrm{N}}(A), A$ is common knowledge for the whole group, which holds if for all strings $x$ of knowledge operators, $x A$ holds. We shall give now the precise definition of the level of knowledge, but first let's look at the set $T(A, H)$ of all strings of knowledge operators $x$ such that $x A$ is true in $H$ :

$$
T(A, H)=\left\{x \mid x \in \Sigma^{*} \text { and } H \vDash x A\right\} .
$$

If there is some non-empty string $x_{0}$ in $T(A, H)$, then $T(A, H)$ is infinite. This is a consequence of the following theorem: 
Theorem 1. (a) Let $\Sigma$ be the alphabet whose symbols are $\left\{K_{1}, \ldots, K_{n}\right\}$. For all $a$ in $\Sigma$, and for all $x, y$, in $\Sigma^{*}$, and all formulae $A$,

$$
\vdash x a y A \leftrightarrow x a a y A,
$$

and hence for all $H, H \vDash x a y A$ iff $H \vDash x a a y A$. That is, repeated occurrences of a are without effect and if $x a y \in T(A, H)$ then $\forall n x a^{n} y \in T(A, H)$.

(b) For a subset $U$ of $\{1, \ldots, n\}$, let $\Delta=\left\{K_{i} \mid i \in U\right\}$. Then for all histories $H$, and formulae $A, H \vDash C_{U}(A)$ iff for all strings $x \in \Delta^{*}, H \vDash x A$.

Proof. (a) is straightforward using the fact that $\approx_{i}$ is an equivalence relation. Let $a=K_{i}$. Then $H \vDash a y A$ iff $\forall H^{\prime}, H^{\prime} \approx_{i} H, H^{\prime} \vDash y A$. But by transitivity of $\approx_{i}$ this yields: $\forall H^{\prime}, H^{\prime} \approx_{i} H, \forall H^{\prime \prime}, H^{\prime \prime} \approx_{i} H^{\prime}, H^{\prime \prime} \vDash y A$, i.e. $H \vDash$ aay $A$. Since $a y A$ and aay $A$ are equivalent, it is easily seen that so are $x a y A$ and $x a a y A$. The proof of (b) follows from the fact that the relation $\approx_{\Delta}$ is the transitive closure of $\cup\left(\approx_{i}\right): i \in \Delta$.

Since occurrences of substrings of the form $K_{i} K_{i}$ don't carry any more information than strings $K_{i}$ we define levels of knowledge of formulae by excluding all strings containing consecutive occurrences of the same knowledge operator.

\section{DEFINITION 1}

(a) A string $x$ is simple if $x$ contains no substrings $K_{i} K_{i}$.

(b) Given a formula $A$ and a history $H$, the level $L(A, H)$ of $A$ at $H$ is the set of all simple $x$ in $\Sigma^{*}$ such that $H \vDash x A$.

If $H$ is clear from the context, or not important, then we shall drop it as a parameter. The set of simple strings on an alphabet $\Sigma$ will be denoted $\Sigma^{s}$. Thus $L(A, H)$ will always be a subset of $\Sigma^{s}$.

\subsection{Embeddability}

Now we will try to characterize levels of knowledge. First we need to introduce the embeddability ordering on strings which turns out to be important.

\section{DEFINITION 2}

Given two strings $x$ and $y$, we say that $x$ is embeddable in $y(x \leqslant y)$, if all the symbols of $x$ occur in $y$, in the same order, but not necessarily consecutively. Formally: Let $x=a_{1} \cdots a_{m}$ and $y=b_{1} \cdots b_{p}$. Then $x$ is embeddable in $y$ iff there is a function $f$ from $\{1, \ldots, m\}$ to $\{1, \ldots, p\}$ such that $\forall i<j \leqslant m, f(i)<f(j)$ and $a_{i}=b_{f(i)}$.

Thus the string $a b a$ is embeddable in itself, in $a a b a$ and in $a b c a$, but not in $a a b b$.

Properties of the embeddability relation $\leqslant$

Fact 1. Embeddability is a well partial order, i.e. it is not only well founded, but every linear order that extends it is a well order (equivalently, it is well founded and every set of mutually incomparable elements is finite). 
Fact 2. Embeddability can be tested in linear time by a two tape Turing machine.

For a proof of fact 1, see Higman (1952) and deJongh \& Parikh (1977). Fact 2 is straightforward.

We also need a weaker (larger) relation defined on $\Sigma^{*}$, which we call $K$-embeddability.

\section{DEFINITION 3}

We define the $K$-embeddability relation $\preccurlyeq$ as follows:

If $x=a_{1} \cdots a_{m}$ and $y=b_{1} \cdots b_{p}$ are elements of $\left\{K_{1}, \ldots, K_{n}\right\}^{*}$, then $x$ is $K$-embeddable in $y$ iff there is a function $f$ from $\{1, \ldots, m\}$ to $\{1, \ldots, p\}$ such that $\forall i \leqslant j \leqslant m, f(i) \leqslant f(j)$ and $a_{i}=b_{f(i)}$.

The condition defining $\preccurlyeq$ is weaker than that defining $\leqslant$. Hence the relation $\preccurlyeq$ extends the relation $\leqslant$ so that $a a b a \preccurlyeq a b a$, but $a a b a \leqslant a b a$. However, for all simple $x$ and for all $y, x \leqslant y$ iff $x \leqslant y$. Given a string $x$, there is a shortest simple $y$ such that $x \preccurlyeq y$. We shall denote $y$ as $\operatorname{Sim}(x)$, the simplification of $x$. For example, if $x=a b b a c b b a a$ then $\operatorname{Sim}(x)=a b a c b a$.

\section{DEFINITION 4}

A downward closed subset of $\Sigma^{s}=\left\{K_{1}, \ldots, K_{n}\right\}^{s}$ is a subset $X$ such that if $x \in X$ and $y \preccurlyeq x$, then $y \in X$.

Theorem 2. For all strings $x \leqslant y \in \Sigma^{s}$, all formulae $A$ and for all histories $H$, if $H \vDash y A$ then $H \vDash x A$.

Proof. We use induction on the sum of lengths of $x$ and $y$. If the sum is 0 , then the lemma is immediate. Otherwise $y$ must be nonempty and let $y$ be $K_{i} y^{\prime}$. Now either $x \preccurlyeq y^{\prime}$ or $x$ is $K_{i} x^{\prime}$ for some $i$ and $x^{\prime} \preccurlyeq y^{\prime}$. In the first case $y A$ implies $y^{\prime} A$ which (by induction hypothesis) implies $x A$. In the second case, $y^{\prime} A$ implies $x^{\prime} A$ by induction hypothesis, and therefore by necessitation $K_{i} y^{\prime} A$ implies $K_{i} x^{\prime} A$, so we get $y A$ implies $x A$.

\section{COROLLARY 1}

Every level of knowledge is a set of simple strings, downward closed with respect to the order $\preccurlyeq$.

\section{COROLLARY 2}

The complement of every level of knowledge is upward closed with respect to $\preccurlyeq$.

So far we have a necessary condition for the set of strings of knowledge operators to be the level of knowledge of some formula in some history. We can infer for example that there is no formula $A$ and history $H$, such that $H \vDash K_{2} K_{1} A$ and $H \vDash \neg K_{2} A$. This is because if $K_{2} K_{1} \in L(A, H)$ then since $L(A, H)$ is downward closed, $K_{2}$ is also in it. In this case, we could also have seen this fact directly by deriving $K_{2} A$ from $K_{2} K_{1} A$, but other cases might be more subtle. Thus we will need the notion of the smallest downward closed set of strings of knowledge operators including the given set $X$. We will call this set the downward closure of $X$ and denote it as $d c(X)$. We start by investigating some properties of the operation of downward closure. 


\subsection{Downward closures}

\section{DEFINITION 5}

The downward closure $d c(X)$, of $X \subseteq \Sigma^{s}$ is the smallest set $Y \subseteq \Sigma^{s}$ such that $X \subseteq Y$ and for all $x$, if $x \in Y$ and $y \preccurlyeq x$ then $y \in Y$.

Properties of downward closure

Facts 3 and 4 depend only on the fact that $\preccurlyeq$ is a partial order.

Fact 3. If $B$ is downward closed then for all $A, A \subseteq B$ iff for every $x \in A$ there is $y \in B$ such that $x \leqslant y$.

Fact 4. $\quad d c(A \cup B)=d c(A) \cup d c(B)$

Fact 5. $d c(A ; B)=\operatorname{Sim}(d c(A) ; d c(B))$ where, for $X \subseteq \Sigma^{*}, \operatorname{Sim}(X)=\{\operatorname{Sim}(x) \mid x \in X\}$. Obviously $\operatorname{Sim}(X) \subseteq \Sigma^{s}$.

In future, we will omit the operator Sim in contexts where non-repeating strings are the only strings involved, stipulating that for subsets $X, Y$ of $\Sigma^{s}, X ; Y$ will indicate $\operatorname{Sim}(X ; Y)$ where the second ';' denotes concatenation of $X, Y$ as subsets of $\Sigma^{*} . A^{s}$ will denote $\operatorname{Sim}\left(A^{*}\right)$.

Fact 6. $\operatorname{dc}\left(A^{s}\right)=U^{s}$ where $U=\{\sigma \mid \sigma \in \Sigma, \exists x \in A \sigma \preccurlyeq x\}$.

Facts 3, 4 and 5 are straightforward. To prove fact 6 first notice that for any $U \subseteq \Sigma$, $U^{s}$ is downward closed, and clearly $d c\left(A^{s}\right) \subseteq U^{s}$. To show that $U^{s} \subseteq d c\left(A^{s}\right)$ let's assume that in fact there is some string $x \in \Sigma^{s}$, such that $x \in U^{s}$ and $x \notin d c\left(A^{s}\right)$. Say $x=a_{1} a_{2} \cdots a_{n}$, where for every $a_{i}, a_{i} \in \Sigma$ and there is $x_{i} \in A$, such that $a_{i} \leqslant x_{i}, x_{1} x_{2} \cdots x_{n} \in A^{n}$, therefore $x_{1} x_{2} \cdots x_{n} \in A^{s}$. Clearly $d c\left(\left\{x_{1} x_{2} \cdots x_{n}\right\}\right) \subseteq d c\left(A^{s}\right)$, but by fact $5, d c\left(\left\{x_{1} x_{2} \cdots x_{n}\right\}\right)=$ $d c\left(\left\{x_{1}\right\}\right) \cdots d c\left(\left\{x_{n}\right\}\right)$. So since $a_{i} \leqslant x_{i}$ for all $i=1, \ldots, n$ then $a_{i} \in d c\left(\left\{x_{i}\right\}\right)$ for all $i=1, \ldots, n$, so $x=a_{1} a_{2} \cdots a_{n} \in d c\left(\left\{x_{1} x_{2} \cdots x_{n}\right\}\right) \subseteq d c\left(A^{s}\right)$ and we get a contradiction.

Fact 7. If $a x \preccurlyeq b y$ and $a \neq b$ then $a x \preccurlyeq y$.

\subsection{Characterization of levels of knowledge}

Now we look at the possibility of characterization of levels of knowledge.

Let $L(A, H)=L(A)$ be a level of knowledge. Let $\bar{L}(A)$ denote the complement of $L(A)$ with respect to $\Sigma^{s}$. Then $\bar{L}(A)$ is upward closed and under each element $y$ of $\bar{L}(A)$ there is a minimal element $x$. Let $m(A)=\left\{x_{1}, \ldots, x_{k}\right\}$ be the set of minimal elements of $\bar{L}(A)$. Then the elements of $m(A)$ are mutually incomparable and since $\preccurlyeq$ is a well partial ordering, $m(A)$ is finite. Now we get:

$$
\bar{L}(A)=\{y \mid \exists x \in m(A) x \preccurlyeq y\} \quad \text { i.e. } L(A)=\{y \mid \forall x \in m(A) x \leqslant y\}
$$

Thus the level of $A$ is completely characterized by the finite set $m(A)$ and we get the next theorem.

Theorem 3. There are only countably many levels of knowledge and in fact all of them are regular subsets of $\Sigma^{s}$ (or $\Sigma^{s}$ ).

Proof. Since $m(A)$ is finite, a finite automaton can clearly be designed to test whether $x \leqslant y$ holds for some element $x$ of $m(A)$, where $y$ is the input. The fact that there are only countably many levels of knowledge follows immediately. 


\section{COROLLARY 1}

The membership problem for a level of knowledge is solvable in linear time.

For a set $L(A)$ we could also look at the set of all maximal elements of $L(A)$ (with respect to $\preccurlyeq)$. Clearly these maximal elements are also mutually incomparable. However, the set $L(A)$ need not be characterized by them since if $A$ is common knowledge $\left(L(A)=\Sigma^{s}\right)$ then the set of maximal elements is empty. The set of maximal elements is also empty if $L(A)=\Delta^{s}$ where $\Delta$ is a proper subset of $\Sigma$. Since distinct sets $L(A)$ can have the same maximal elements, maximal elements cannot characterize $L(A)$.

However, with finite levels, maximal elements do in fact characterize $L(A)$.

Theorem 4. If $L$ is a non-empty finite subset of $\Sigma^{s}$, then $L$ is downward closed iff for some $k$ and $x_{i}: i \leqslant k$ where $x_{i} \in \Sigma^{s}$,

$$
L=\bigcup_{i=1}^{k} d c\left(\left\{x_{i}\right\}\right)
$$

Proof. Clearly if $L$ is a union of downward closed sets, then by fact $4, L$ is downward closed.

Conversely, let $L=\left\{x_{1}, \ldots, x_{m}\right\}$ be a finite downward closed set.

Consider $L^{\prime}=\cup_{i=1}^{m} d c\left(\left\{x_{i}\right\}\right)$. Clearly $L \subseteq L^{\prime}$. Let $x \in L^{\prime}$. Then there is $i$ such that $x \in d c\left(\left\{x_{i}\right\}\right)$. Since $x_{i} \in L$ and $L$ is downward closed, then $x \in L$. So $L=L^{\prime}$ and therefore $L^{\prime}$ is the required representation of $L$.

Remark. Note that we could have taken just the maximal elements of $L$ and they would be mutually incomparable in that case. If two finite sets $L(A), L\left(A^{\prime}\right)$ have the same maximal elements, they must coincide.

In order to analyse infinite levels, we first need to establish some properties of downward closed sets. The following theorem generalizes the representation from the previous theorem to the case of infinite levels. It will be used in obtaining a "normal form" theorem for the levels of knowledge.

\section{DEFINITION 6}

A subset $L$ of $\Sigma^{s}$ is star-linear iff there exist strings $x_{1}, \ldots, x_{m+1}$ and subsets $\Delta_{1}, \ldots, \Delta_{m}$ of $\Sigma$ such that $L=d c\left[\left(\left\{x_{1}\right\}\right) \Delta_{1}^{*}\left(\left\{x_{2}\right\}\right) \Delta_{2}^{*} \cdots \Delta_{m}^{*}\left(\left\{x_{m+1}\right\}\right)\right] \cap \Sigma^{s}$.

Theorem 5. If $L$ is a subset of $\Sigma^{s}$, then $L$ is downward closed iff $L$ is a finite union of star-linear sets.

Proof.

$\leftarrow$

Star-linear sets are downward closed, so by fact 4 , so is $L$.

$\Rightarrow$

$L$ is a downward closed subset of $\Sigma^{s}$, so $L$ is regular and $L$ is the language accepted by some finite automaton $M(L=L(M))$. 
Let $S$ be the set of all states of $M$. Let $s \stackrel{x}{\rightarrow} t$ iff there is a sequence of transitions from the state $s$ to the state $t$ labeled by the symbols forming the string $x$. We define an equivalence relation $\sim$ on $S: s \sim t$ iff $\exists x, y \stackrel{x}{\rightarrow} t$ and $t \stackrel{y}{\rightarrow} s$. Intuitively, two states are $\sim$-equivalent iff they are in the same loop in $M$. Now we define a new automaton $M^{\prime}$, whose states are the equivalence classes $[s]$ of $\sim$. $[s] \stackrel{a}{\rightarrow}[t]$ if there exist $s^{\prime} \in[s]$ and $t^{\prime} \in[t]$ such that $s^{\prime} \stackrel{a}{\rightarrow} t^{\prime}$. We also add transitions $[s] \stackrel{a}{\rightarrow}[s]$ if $\exists s^{\prime}, s^{\prime \prime} \in[s]$ such that $s^{\prime} \stackrel{\sigma}{\rightarrow} s^{\prime \prime}$ in $M$. The accepting states of $M^{\prime}$ are the equivalence classes of accepting states in $M$, and the initial state of $M^{\prime}$ is the equivalence class of the initial state $q_{1}$ of $M$.

It is easy to see that $L(M) \subseteq L\left(M^{\prime}\right)$. We will show that $L^{\prime}\left(M^{\prime}\right) \subseteq L(M)$.

Let $x \in L\left(M^{\prime}\right) . x=a_{1} a_{2} \cdots a_{m} .\left[s_{1}\right] \stackrel{a_{1}}{\rightarrow}\left[s_{2}\right] \stackrel{a_{2}}{\rightarrow}\left[s_{3}\right] \cdots\left[s_{m}\right] \stackrel{a_{m}}{\rightarrow}\left[s_{m+1}\right]$ where $s_{m+1}$ is accepting in $M$ and $s_{1}=q_{1}$ is the initial state.

If $\left[s_{1}\right] \stackrel{a_{1}}{\rightarrow}\left[s_{2}\right]$, there must be states $s_{1}^{\prime}, s_{2}^{\prime}$ such that $s_{1}^{\prime} \in\left[s_{1}\right], s_{2}^{\prime} \in\left[s_{2}\right]$ and $s_{1}^{\prime} a_{4} s_{2}^{\prime}$ in $M$.

Let $y_{1}, y_{2}$ be strings such that $s_{1} \stackrel{y_{1}}{\rightarrow} s_{1}^{\prime}, s_{2}^{\prime} \stackrel{y_{2}}{\rightarrow} s_{2}$, so we have a string $z_{1}=y_{1} a_{1} y_{2}$ for which $s_{1} \stackrel{z_{1}}{\rightarrow} s_{2}$ in $M$. Repeating this procedure we can find strings $z_{2}, \ldots, z_{k}$ such that $s_{1} \underline{z}_{4} s_{2} \stackrel{z_{2}}{\rightarrow} s_{3} \cdots s_{m}{ }_{m} \rightarrow s_{m+1}$ and for all $j \leqslant k, a_{j} \leqslant z_{j}$. Therefore $z=z_{1} z_{2} \cdots z_{m} \in L(M)\left(s_{m+1}\right.$ is accepting in $M$ ) and since $L(M)$ is downward closed and $x \leqslant z, x \in L(M)$.

We have shown that $L(M)=L\left(M^{\prime}\right)$. We show now that the latter has the required form.

The only loops in $M^{\prime}$ are of the form $[s] \stackrel{a}{\rightarrow}[s]$. A state $[s]$ is cycling if there is a $\sigma$ such that $[s] \stackrel{\sigma}{\rightarrow}[s]$.

Let $B_{i}$ be a sequence $\left[s_{1}\right] a_{1}\left[s_{2}\right] a_{2} \cdots\left[s_{l+1}\right]$ from the initial state $\left[s_{1}\right]=\left[q_{1}\right]$ of $M^{\prime}$ to an accepting state $[t]=\left[s_{l+1}\right]$, where the $a_{j}$ are symbols such that $\left[s_{j}\right] \stackrel{a_{j}}{\rightarrow}\left[s_{j+1}\right]$, and $\left[s_{j}\right] \neq\left[s_{j+p}\right]$ for any $p$ (there are no loops in the sequence). With this sequence we associate a star-linear set $\operatorname{Sim}(X)=d c\left[\Delta_{1}^{s} a_{1} \Delta_{2}^{s} \cdots a_{l} \Delta_{l+1}^{s}\right] \cap \Sigma^{s}$ where, for all $i$, if $\left[s_{i}\right]$ is non-cyclic, then $\Delta_{i}=$ null and otherwise, $\Delta_{i}=\left\{\sigma \mid\left[s_{i}\right] \stackrel{G}{\rightarrow}\left[s_{i}\right]\right\}$. Then the set of nonrepeating strings that take us from $\left[s_{1}\right]$ to $[t]$ is exactly $X$.

If we take the union of all such sets $X$ (there are finitely many of them), then we get the characterization of $L\left(M^{\prime}\right)$ in the required form.

We have found a representation of downward closed sets, and therefore of levels of knowledge. We prove now that there is a unique minimal representation of such a form.

Theorem 6. Every star-linear set $X$ is directed with respect to the embeddability relation $\preccurlyeq$. In other words: for all $x^{\prime}, x^{\prime \prime} \in X$ there is $x \in X$ such that $x^{\prime} \leqslant x$ and $x^{\prime \prime} \preccurlyeq x$.

Proof. Let $x^{\prime}=y_{1} v_{1} \cdots y_{m} v_{m} y_{m+1}$ and $x^{\prime \prime}=z_{1} w_{1} \cdots z_{m} w_{m} z_{m+1}$ where $y_{i}, z_{i} \in d c\left(\left\{x_{i}\right\}\right)$, and $v_{i}, w_{i} \in \Delta_{i}^{s}$ for $i=1, \ldots, m$. Then if we take $x=x_{1} v_{1} w_{1} \cdots x_{m} v_{m} w_{m} x_{m+1}$, then clearly $x^{\prime} \preccurlyeq x, x^{\prime \prime} \leqslant x$ and of course $x \in X$.

Theorem 7. If $X \subseteq \cup_{j=1}^{l} Y_{j}$, where $X, Y_{j}$ are all star-linear, then there exists a $j$ such that $X \subseteq Y_{j}$.

Proof. Suppose that $X \subseteq \cup_{j=1}^{l} Y_{j}$ and for all $j, X \nsubseteq Y_{j}$, then there are $x_{j} \in X$, $j=1, \ldots, l$, such that $x_{j} \notin Y_{j}$. By theorem 6 applied $l-1$ times, we can find $x \in X$ such that for all $j, x_{j} \preccurlyeq x$. Since all $Y_{j}$ are downward closed, $x_{j} \preccurlyeq x$ and $x_{j} \notin Y_{j}$, so $x$ is not in any $Y_{j}$. Thus $x \notin \cup_{j=1}^{l} Y_{j}$, but $x \in X$ and we get a contradiction. 
Theorem 8. If $L$ is downward closed then $L$ has a unique representation as a finite minimal union of star-linear sets.

Proof. Clearly we have a minimal representation of $L, L=\cup_{i=1}^{k} X_{i}$ and suppose we have some other representation of $L$ of the same form $L=\cup_{j=1}^{l} Y_{j}$, where all $X_{i}$ and $Y_{j}$ 's are star-linear sets. Then for every $i, X_{i} \subseteq \cup_{j=1}^{l} Y_{j}$. So by theorem 7 , for every $X_{i}$ there is $Y_{j}$ such that $X_{i} \subseteq Y_{j}$. Similarly, by applying theorem 7 to $Y_{j}$ we get some $m$ such that $Y_{j} \subseteq X_{m}$, so we have for every $i$, some $j, m$ such that $X_{i} \subseteq Y_{j} \subseteq X_{m}$. By minimality of the $\left\{X_{i}\right\}_{i=1, \ldots, k}$ representation $i=m$, so we have $X_{i} \subseteq Y_{j} \subseteq X_{i}$ and hence $X_{i}=Y_{j}$. Similarly, every $Y_{j}$ equals some $X_{i}$ and the two representations are the same.

COROLLARY (Normal form theorem)

Every level of knowledge $L$ of a formula $A$ in a distributed system has a unique representation as a finite minimal union of star-linear sets.

\subsection{Representation of levels of knowledge using minimal strings of the complement}

It turns out that the representation of levels of knowledge using star-linear sets is convenient if we want to realize at least a given level of knowledge and if we have both synchronous and asynchronous communications in the system. It is not appropriate if we want to realize at most a given level and if we have only synchronous communications in the system. In such cases the following theorem is useful:

\section{DEFINITION 7}

Given strings $x_{1}, \ldots, x_{k}$, not containing repetitions, let $N\left(x_{1}, \ldots, x_{k}\right)$ be the set $\left\{y \mid \forall i \preccurlyeq k, x_{i} \nless y\right\}$.

\section{Theorem 9}

(a) $N\left(x_{1}, \ldots, x_{k}\right)=\cap N\left(x_{i}\right): i \leqslant k$.

(b) If $x=a_{1} \cdots a_{m}$ then $N(x)=\left(\Sigma-a_{1}\right)^{s} \cdots\left(\Sigma-a_{m}\right)^{s}$.

Proof. The first part is obvious.

To see (b) we use induction on $m$.

First of all, it is clear that $N(x)$ includes the right hand side. For suppose $y \in\left(\Sigma-a_{1}\right)^{s} \cdots\left(\Sigma-a_{m}\right)^{s}$. If $y$ has no $a_{1}$, then it is certainly in $N(x)$. Else the first occurrence of an $a_{1}$ in $y$ must be from the $\left(\Sigma-a_{2}\right)^{s}$ at the earliest. $y$ is then of the form $y_{1} a_{1} y_{2}$ where $y_{1}$ contains no $a_{1}$ and $y_{2}$ is in $\left(\Sigma-a_{2}\right)^{s} \cdots\left(\Sigma-a_{m}\right)^{s}$. By induction hypothesis $a_{2} \cdots a_{m} y_{2}$ and so $x \leqslant y$.

Suppose now that $y$ is in $N(x)$. We want to show that $y \in\left(\Sigma-a_{1}\right)^{s} \cdots\left(\Sigma-a_{m}\right)^{s}$. If $m=1$ then this is immediate. Otherwise we know that $x \neq y$.

If $a_{1} \nless y$, then $y \in\left(\Sigma-a_{1}\right)^{s}$ and hence $y \in\left(\Sigma-a_{1}\right)^{s} \cdots\left(\Sigma-a_{m}\right)^{s}$.

If $a_{1} \preccurlyeq y$ then $y=y_{1} a_{1} y_{2}$, where $a_{1} \nless y_{1}$ and $a_{2} \cdots a_{m} \nless y_{2}$. In that case $y_{2} \in\left(\Sigma-a_{2}\right)^{s} \ldots$ $\left(\Sigma-a_{m}\right)^{s}$ by the induction hypothesis and $y_{1} \in\left(\Sigma-a_{1}\right)^{s}$. Since $a_{1} \neq a_{2}$, the result follows. 


\section{Model of a distributed system}

We assume that there are a finite number of processes, $1, \ldots, n$, which compute and communicate with each other either by asynchronous messages or by broadcasts. Our network is assumed to be fully connected ${ }^{3}$ (there is a channel from every process to every other process).

Asynchronous communication consists of two phases: send and receive. All messages sent are ultimately delivered (and in the order in which they were sent) but the delay (transmission time) may be arbitrarily long.

Broadcasts are fully reliable, synchronous communications ${ }^{4}$ where all processes involved simultaneously receive the message sent by one of them. Our broadcasts are therefore similar to CSP communication, but allow for more then two particpants at a time. Later on we will point out a limitation of CSP that follows from our results.

We consider three kinds of systems - systems where only asynchronous communication is available, systems where only synchronous communication is available and systems where both kinds of communication are available.

There is assumed to be a global clock in the background which orders all events, but this clock is not assumed to be available to the processes. Time is discrete, the clock is set to 0 at the beginning of every computation and grows in increments of one.

In order for the processors to have something to communicate, we ensure that for every processor in our system there are facts known initially only to this processor. To accomplish that we assume that every process starts its computation with some initial value, a finite string of 0 's and 1's. This initial value may correspond to some local non-deterministic input, e.g. the result of a sequence of coin tosses by a processor.

At each moment of global time, zero or more events may take place, at most one at each process. This finite set of local events constitutes a global event and a global history is simply a possible sequence of global events. What global histories are possible is determined by the programs of the various individual processes as well as by the properties of the means of communication. The protocol $\mathbf{P}$ is just the set of all possible global histories, closed under the prefix operation.

Different models of distributed systems have been used by different authors. Our model is similar to that used by Parikh \& Ramanujam (1985, pp. 256-268), Halpern \& Moses (1990) and by Chandy \& Misra (1986).

There are, however, some small differences: we assume that there is no global clock in the system accessible to the processes ("common clock" in Parikh \& Ramanujam 1985, pp. 256-268). Parikh \& Ramanujam (1985, pp. 256-268) do not have this

\footnotetext{
${ }^{3}$ If the network is not fully connected then some levels of knowledge may be impossible to realize due to the lack of communication capabilities, e.g. if a processor is isolated (cannot communicate with anyone) then the other processes cannot learn anything from that process. Interesting questions arise in case of a directed network where every process may communicate with every other process but some communications are necessarily indirect (go through other processes). We will not analyse this case here.

${ }^{4}$ The two kinds of communications can be thought of as two kinds of communication media e.g. mailing system (asynchronous) and telephone lines (synchronous). Since we allow for synchronous communication between more than two processes at a time, our telephone system must have "conference call" capability. Note that the "handshake' in CSP can be thought of as a special case of a broadcast involving exactly two processes.
} 
restriction. In their terminology, our local histories are always individual views of "time-free" global histories.

Chandy \& Misra (1986) do not allow different events to occur locally in different sites at the same instant of time. We shall not impose this restriction. This can be interpreted in two ways: either we can treat time in our system as "less refined" than in Chandy \& Misra (1986), or we can interpret our system as synchronous, events in all sites being governed by the same global clock. Differences in local time will then arise solely from the fact that a process may not have an event happening at every moment of global time. If a process is inactive in a certain round (its action is the "null" event), it cannot perceive that time has passed. Hence even while assuming that our system runs in synchronous rounds, we ensure that processes are not able to record global time. Consequently they cannot draw any conclusions about the others merely by observing their own local clock.

There are some terminological differences between our description of a distributed system and that of Halpern \& Moses. Following Parikh \& Ramanujam (1985, pp. 256-268), we shall denote the set of all global histories (runs in Halpern \& Moses $1990)$ as the protocol. Halpern \& Moses (1990) use the word protocol to describe the rules governing actions of processors (effectively generating the set of runs).

\subsection{Definitions}

Here we formally specify our class of models. Let $N=\{1, \ldots, n\}$ be the set of all processors. Every processor $i$ has infinitely many possible initial states $v$ and an initial state is a string of 0 's and 1 's $\left(v \in\{0,1\}^{*}\right)$. We denote the set of initial states for $i$ by $V_{i}$. The set of global initial states is $\mathscr{V}=\Pi_{i=1}^{n} V_{i}$.

From now on we will use lower case letters to denote everything pertaining to a single process. Capitals without subscripts will be used where all the processes are involved (e.g. $v_{i}$ is an initial state of a processor $i$, while $V$ is an initial configuration of the whole system: $\left.V=\left(v_{1}, \ldots, v_{n}\right)\right)$. $\mathscr{V}$ is the set of all such $V . v_{i}$, initial input of the processor $i$ provides interpretation for private facts in our logic, i.e. $Q_{i, m}$ is true iff the $m$ th bit of $v_{i}$ is 1 . The $m$ th private fact $P_{i, m}$ of a processor $i$ is whether $Q_{i, m}$ or its negation is true.

Events. $E_{i}$ denotes the set of all events in which processor $i$ can participate (events local to $i$ ). There are the following types of events (or actions):

(1) $L_{i}$ : Local computation steps. (We assume that for $i \neq j, L_{i} \cap L_{j}=\phi$ )

(2) $s(i, j, m)$ : Sending a message $m$ to a processor $j, j \in N$.

(3) $r(i, j, m)$ : Receiving a message $m$ from a processor $j, j \in N$.

(4) $b c(i . U, m)$ : Sending a broadcast $m$ to a group of processors $U, i \in U \subseteq N$. The same event is also in $E_{j}$ for all $j$ in $U$.

$$
\begin{aligned}
& E_{i}=L_{i} \cup\{s(i, j, m) \mid m \in M, j \in N\} \cup\{r(i, j, m) \mid m \in M, j \in N\} \\
& \cup\{b c(j, U, m) \mid m \in M, i, j \in U \subseteq N\} \cup\{b c(i, U, m) \mid m \in M, i \in U \subseteq N\}
\end{aligned}
$$

Note that the last two items can be combined into one as $\{b c(j, U, m) \mid m \in M, i \in U \subseteq N\}$. $M$ is the set of messages, defined below.

We define the set of global events $\mathbf{G}$ in our system. $\mathbf{G} \subseteq \Pi_{i=1}^{n}\left(E_{i} \cup\{\right.$ null $\}$ ) (a cartesian product) such that if $\left(e_{1}, \ldots, e_{i}, \ldots, e_{n}\right) \in \mathbf{G}$ for some $i$ and $e_{i}=b c(j, U, m)$ then 
for all $i^{\prime} \in U, e_{i^{\prime}}=b c(j, U, m)$. If $e_{i}=$ null for some $i$, it means that there is no local event at $i$ at this point. Note that null is not local to any process. We use the notation $(G)_{i}$ to denote the $i$ th coordinate of $G$, so $\left(e_{1}, \ldots, e_{i}, \ldots, e_{n}\right)_{i}=e_{i}, \ldots, e_{i}$.

Histories. A history (a run) is an input value followed by a sequence of events. The set of all possible histories of the system will be called the protocol $\mathbf{P}$. So $\mathbf{P} \subseteq \mathscr{V} ; \mathbf{G}^{*}$. Protocols are always closed under taking an initial segment of a history: $H \in \mathbf{P}$ implies that every $H^{\prime}$ which is an initial segment of $H$ is in $\mathbf{P}$.

We will require that for every receive in every history in every protocol, there is exactly one corresponding send, which occurs before the receive (this condition will be called time-consistency).

We say that two histories $H$ and $H^{\prime}$ are compatible iff they start with the same input values (initial states).

We define the concatenation of compatible histories:

If $H_{1}=V ; G_{1} ; \ldots ; G_{k}$, and $H_{2}=V ; G_{1}^{\prime} ; \ldots ; G_{l}^{\prime}$, then the concatenation of $H_{1}$ and $H_{2}$ is the history $H=V ; G_{1} ; \ldots ; G_{k} ; G_{1}^{\prime} ; \ldots ; G_{l}^{\prime}$.

Local histories are the projections of global histories onto the sets of local events of the processors. They are "time-forgetting", i.e. they erase null events.

We assume that a global event - the ticking of the clock - takes place even if no local events take place at a particular moment (this corresponds to events of the form (null, ..., null)), and so the length of a global history is just the amount of time elapsed. However, what each process sees at any moment of time is its local event, if any, and its local history is simply the sequence of local events. Given $i$, and the global history $H$, the local history $h_{i}$ is uniquely defined and we let $\Phi_{i}$ be the map which takes us from $H$ to $h_{i}$.

We can inductively define $\Phi_{i}$ 's as follows:

$$
\begin{aligned}
\Phi_{i}\left(\left(v_{1}, \ldots, v_{n}\right)\right) & =\left(v_{1}, \ldots, v_{n}\right)_{i}=v_{i}, \\
\Phi_{i}(H ; G) & = \begin{cases}\Phi_{i}(H) ; e_{i}, & \text { iff }(G)_{i}=e_{i} \in E_{i}, \\
\Phi_{i}(H), & \text { iff }(G)_{i}=\text { null } .\end{cases}
\end{aligned}
$$

So $\Phi_{i}$ is like ()$_{i}$ (when we extend the projection operation from events to histories) except that it erases null events.

Its local history is all that a processor sees, so all global histories which correspond to the same local history $h_{i}$ look the same to the processor $i$. Note that the length of $\Phi_{i}(H)$ is less than or equal to the length of $H$. In fact length $\left(\Phi_{i}(H)\right)=\operatorname{length}(H)$ iff there are no null events on $i$ in $H$.

For every $i$ we can define an equivalence relation on the set of global histories:

$$
H \approx_{i} H^{\prime} \text { iff } \Phi_{i}(H)=\Phi_{i}\left(H^{\prime}\right)
$$

If $U$ is a subset of $N$, then we let $\approx_{U}^{*}$ be the reflexive transitive closure of $U \approx_{i}: i \in U$.

We use capital letters to denote global histories, events etc., lower case letters to denote local histories, events etc.

Time. The global time of an event $G$ in a global history $H$, Time $(G, H)$, is the length of the initial segment of $H$ up to and including $G$, the time when the event $G$ has occurred in the history $H$.

Note that since null $\notin E_{i}$ for all $i$, processes do not have access to the global clock. 
They can introduce their own local logical clocks, but these clocks do not have to coincide. Local time of $e_{i}$ in $h_{i}$ can be defined as time $\left(e_{i}, h_{i}\right)-$ the length of $h_{i}$ up to and including $e_{i}$.

The lack of the access to the global clock, together with the closure conditions for the protocol guarantee that the only possible "causality" ordering that can be defined corresponds in case of asynchronous systems to Lamport's (1978) "happened before" ordering 5 .

Messages. Messages $m$ are knowledge formulae (formulae of the form $x A$ where $x$ is a string of knowledge operators $K_{i_{1}} K_{i_{2}} \cdots K_{i_{n}}$ and $A$ is some boolean combination of $P_{1}, \ldots, P_{n}$ ). We assume that the processes are "honest", they only send (or broadcast) messages which they know are true. Formally, if $\Phi_{i}(H)=h_{i} ; s(i, j, B)$ or $\Phi_{i}(H)=h_{i}$; $b c(i, U, B)$ and $H \in \mathbf{P}$, then if $\Phi_{i}\left(H^{\prime}\right)=h_{i}$ then $H^{\prime} \vDash K_{i} B$. Later on we will also allow certain 'common knowledge' symbols $C_{U}$ to occur in addition to the $K_{i}$.

We remark that the notion of a message and that of a history should really be defined via mutual recursion since the truth of a message depends on the histories that are possible and the histories that are possible can contain only true messages. However, for the messages of the type we have described, the truth of a message depends only on the portion of the history that has already elapsed. Hence the recursion is allowable. If the messages were to contain future modalities, then there could be problems, which arise due to circularity.

We illustrate our semantics by means of an example. Suppose that for $i \leqslant 3, P_{i}$ is a private fact of process $i$. Suppose also that in a history $H, P_{i}$ are all true. Now process 3 receives a message from process 2 that $P_{1} \rightarrow P_{3} .3$ knows that the message is correct because $P_{3}$ is in fact true. However, 3 has never sent any messages to anyone at all. Now 3 can reason, " 2 cannot know that $P_{3}$ is true so 2 must know that $P_{1}$ is false." We show how to do this argument in our framework. If we replace $H$ by $H^{\prime}$ where $P_{3}$ is false, but otherwise the same as $H$, then $H \approx_{2} H^{\prime}$. Now 2 must have sent in $H$ only a message that he knew to be true, so we must also have $H^{\prime} \vDash K_{2}\left(P_{1} \rightarrow P_{3}\right)$. Hence $H^{\prime} \vDash\left(P_{1} \rightarrow P_{3}\right)$ so that $H^{\prime} \vDash \neg P_{1}$. Since we only used the fact that $H \approx_{2} H^{\prime}$, we get $H \vDash K_{2}\left(\neg P_{1}\right)$. However, this argument could be applied to any $H^{\prime \prime} \approx_{3} H$ so that we get $H \vDash K_{3}\left(K_{2}\left(\neg P_{1}\right)\right)$.

Closure conditions for the protocol. We impose some additional conditions on the protocol $\mathbf{P}$. We want to ensure that the initial state of $i,\left(v_{i}\right)$ cannot be known to any other process $j$ at any run of the system, unless $j$ learns about $v_{i}$ from some communication. We want to exclude the possibility that something may be known "accidentally". To achieve that we will make sure that all initial states are possible. Moreover, if $v_{i}$ is the initial state of $i$, all other strings $v_{i}^{\prime}$ will remain possible for $j$ as initial states of $i$, unless $j$ gets some message to the contrary (directly from $i$ or via some other processors).

(1) All vectors of input values are possible: $\forall V$ such that $V=\left(v_{1}, \ldots, v_{n}\right)$ where every $v_{i}$ is a sequence of 0 's and 1 's, there is some $H \in \mathrm{P}$ such that for some $H^{\prime}, H=V ; H^{\prime}$ (note that $H^{\prime}$ cannot itself be a history since it lacks an input value).

\footnotetext{
${ }^{5} e_{1} \rightarrow e_{2}$ iff $e_{1}$ is send, $e_{2}$ is receive of the same message or $e_{1}, e_{2}$ are local to the same process and $e_{1}$ occurred earlier than $e_{2} . e_{1}$ happened before $e_{2}$ iff $e_{1} \rightarrow^{*} e_{2}$ where $\rightarrow^{*}$ is the reflexive, transitive closure of $\rightarrow$. In a system where we allow broadcast one more condition is needed in the definition of $\rightarrow . e_{1} \rightarrow e_{2}$ if $e_{1}$ and $e_{2}$ are two local projections of the same broadcast.
} 
(2) No sequence of local events on some group of processes can influence possible actions of some other group of processes unless there are some communications (assuming that both groups are disjoint).

For that we need some closure conditions on the set of all protocols. The first condition we use is due to Chandy \& Misra (1986) (it is the first of their principles of computation extension).

We need one definition:

Let $G=\left(e_{1}, \ldots, e_{n}\right), G$ is on $U$ if $U=\left\{i \mid(G)_{i} \neq\right.$ null $\}$ (so $U$ is the set of processes which have some local events in $G$ ).

\section{Closure conditions:}

(i) Extension rule: Suppose that $\forall i \in U, H \approx_{i} H^{\prime}, G$ is on $U$, and none of $(G)_{i}$ is a receive $r(i, j, m)$ for any $j$ not in $U$. Then

$$
H^{\prime} \in \mathbf{P}, \quad H ; G \in \mathbf{P} \Rightarrow H^{\prime} ; G \in \mathbf{P} .
$$

The extension rule guarantees that if we have a protocol $\mathbf{P}$, some history $H$ in $\mathbf{P}$ and some action of a group of processes $U$ is possible in $H$, then the same action must be possible in every history $H^{\prime}$ which looks the same to all processes in $U$ unless it violates time-consistency. In order to see why $e_{i}$ cannot be allowed to be a receive from a processor outside $U$, let us look at an example:

$$
\text { Let } N=\{1,2,3\}, \quad U=\{1,2\} \text {. }
$$

$H=V ;\left(\right.$ null, null, s(3,1,m)), $H^{\prime}=V ;\left(\right.$ null, null, null) for some input $V$. Clearly $H \approx_{1} H^{\prime}$ and $H \approx_{2} H^{\prime}$. If we take $G=(r(1,3, m)$, null, null $)$ such that $H ; G \in \mathbf{P}$ then requiring $H^{\prime} ; G$ to be in $\mathbf{P}$ would violate time-consistency.

The following conditions assure that no process can get any additional information about the other processes by observing its own local events (no hidden synchronization). These conditions are necessary because (unlike Chandy \& Misra 1986) we allow local events at different sites at the same instant of time. Condition (ii) says that if some local events have occurred in parallel, and the sets of participating processes were disjoint, they could have occurred in sequence. We'll call it the splitting rule.

(ii) Splitting rule: $G=\left(e_{1}, \ldots, e_{n}\right), G \notin V, G$ is on $U$. Given $U_{1}, U_{2}$ such that $U_{1} \cup U_{2}=U$ and $U_{1}, U_{2}$ disjoint, then we can "split" $G$ into $G_{1}$ and $G_{2}$ :

$$
H ; G \in \mathbf{P} \Rightarrow H ; G_{1} ; G_{2} \in \mathbf{P},
$$

where $(G)_{i}=\left(G_{1}\right)_{i}$ for $i \in U_{1},(G)_{i}=\left(G_{2}\right)_{i}$ for $i \in U_{2},\left(G_{1}\right)_{j}=$ null $=\left(G_{2}\right)_{k}$ for $j \notin U_{1}, k \notin U_{2}$ provided that we don't split any broadcasts: $(G)_{i}=b c(i, V, m) \rightarrow V \subseteq U_{1} \vee V \subseteq U_{2}$.

Condition (iii) says that if some local events have occurred in sequence, the sets of participating processes were disjoint, and there was no send receive pair in them, they could have occurred in parallel.

(iii) Joining rule: Given $U_{1}, U_{2}$ such that $U_{1} \cup U_{2}=U$ and $U_{1}, U_{2}$ disjoint, let $G_{1}$ be on $U_{1}, G_{2}$ on $U_{2}$, and if there are no $i, j$ such that $\left(G_{1}\right)_{i}=s(i, j, m)$ and $\left(G_{2}\right)_{j}=r(j, i, m)$.

$$
H ; G_{1} ; G_{2} \in \mathbf{P} \Rightarrow H ; G \in \mathbf{P},
$$

where $(G)_{i}=\left(G_{1}\right)_{i}$ for $i \in U_{1},(G)_{i}=\left(G_{2}\right)_{i}$ for $i \in U_{2}$. 
Systems. We consider three kinds of systems. Asynchronous systems are the systems as described above but without broadcasts. The only communications are via send and receive. Synchronous systems are systems in which all communications are done using broadcasts and we do not have the events send and receive. Finally, we use the name mixed communications systems for systems with both kinds of communications available.

\section{Realization of levels of knowledge in distributed systems}

Since we know now that every level of knowledge is a downward closed set of strings, we can ask whether given a downward closed set of strings $L$ we can find some formula $A$ and some run of a distributed system (history $H$ ) such that $L=L(A, H)$. The answer, it turns out, depends on the kind of communication available in the system. In a system with unreliable delivery time (asynchronous system) we are able to realize only finite levels of knowledge (this generalizes the result of Halpern \& Moses (1990) that no common knowledge can be achieved in an asynchronous system). In systems where all communications are instantaneous broadcasts with at least 3 processors synchronous systems - all levels of knowledge can be realized. If there are only 2 processors, and broadcasts are the only medium of communication, then the finite levels containing strings longer then 1 cannot be realized. In the full systems, where there are two communication media: synchronous broadcast, and asynchronous send and receive, all levels can be realized.

So in the following sections we separately analyse these three kinds of systems:

(1) Systems where only asynchronous communications are available.

(2) Systems where only synchronous cummunications are available.

(3) Systems where both synchronous and asynchronous communications are available.

Before we proceed with realizing levels of knowledge, we say something about the properties of the formulae, which we will look at.

\section{DEFINITION 8}

A formula $A$ is persistent if whenever $H \vDash A$ and $H^{\prime}$ extends $H$, then $H^{\prime} \vDash A$.

Theorem 10. If $A$ is persistent then so is $K_{i}(A)$ for any $i$.

Proof. Suppose $H \vDash K_{i}(A)$ and $H^{\prime}$ extends $H$. Suppose that for some $H^{\prime \prime}, \Phi_{i}\left(H^{\prime \prime}\right)$ equals $\Phi_{i}\left(H^{\prime}\right)$. Then for some initial segment $H_{t}$ of $H^{\prime \prime}, \Phi_{i}\left(H_{t}\right)$ equals $\Phi_{i}(H)$. Hence $H_{t}$ satisfies $A$ and by the persistence of $A$, so does $H^{\prime \prime}$. Since $H^{\prime \prime}$ was arbitrary with $\Phi_{i}\left(H^{\prime \prime}\right)=\Phi_{i}\left(H^{\prime}\right), H^{\prime}$ satisfies $K_{i}(A)$.

Theorem 11. Every formula $A$ which is a boolean combination of $P_{i}$ 's is persistent.

\section{COROLLARY 1}

Every formula of the form $x A$, where $A$ is a boolean combination of $P_{i}$ 's, and $x$ is a string of knowledge operators, is persistent. 
From now on we will look only at persistent formulae of the form as in corollary 1 after theorem 11 .

\subsection{Asynchronous case}

We now look at the question of the levels of knowledge in asynchronous systems. The only possible communications are via send and receive, where the arrival time of the message is not guaranteed. In the formal model we exclude broadcasts, so no event is local to more then one processor.

What are the consequences of the fact that messages, although eventually delivered, may remain in the mail for an unbounded amount of time? Suppose that a process $i$ sends a message to a process $j$ that a certain formula $A$ (whose truth value is invariant over time) is true. Then when process $j$ receives the message, it knows $A$ and that $i$ knows $A$. Thus $K_{j}\left(K_{i}(A)\right)$ is true. However, $i$ does not know that $j$ has received the message and hence $K_{i}\left(K_{j}\left(K_{i}(A)\right)\right)$ does not hold until $i$ receives an acknowledgement from $j$.

Let's show that this fact is valid in our model. We'll take only a 2 processor system, the processors are 1 and 2 . Let $I$ be an initial configuration of the system in which the first bit of an input of the first processor is 1 (and this single bit is the whole input of 1$)$. So $I=\left(v_{1}, v_{2}\right), v_{1}=1$. Let $P_{1}$ say that the input value of the processor is 1 , so $P_{1}=Q_{1,1}$. Slightly abusing notation we will write that $I \vDash P_{1}$, instead of $I \vDash P_{1}=Q_{1,1}$. $P_{1}$ is private to 1 . Now let $H$ be a history which starts in the initial configuration $I$ and in which 1 sends a message to 2 informing him that $P_{1}$ and this message is received by 2 in the next instant of time. $H=I ;\left(s\left(1,2, P_{1}\right)\right.$, null $) ;\left(\right.$ null, $\left.r\left(2,1, P_{1}\right)\right)$, and $H \vDash K_{2} K_{1} P_{1}$.

Let $H^{\prime}$ be a history in which the same message is sent, but it is delayed, it is not received by 2 in the second instant of time: $H^{\prime}=I ;\left(s\left(1,2, P_{1}\right)\right.$, null); (null, null).

Because of the extension rule $H^{\prime}$ is in the protocol.

Clearly $H \approx{ }_{1} H^{\prime}$.

Let $H^{\prime \prime}$ be a history with the same input configuration, but in which 1 is slow and hasn't sent anything yet: $H^{\prime \prime}=I$; (null, nul); (null, null) (again, because of the extension rule, $H^{\prime \prime}$ is in the protocol).

$H^{\prime \prime} \approx{ }_{2} H^{\prime}$.

Let $H^{\prime \prime \prime}=I_{1}$; where $I_{1}$ is an initial configuration in which 2 receives the same input as in $H$, but l's input is 0 instead of $1 . H^{\prime \prime \prime}$ is in the protocol because of the first closure condition (all inputs are possible).

$H^{\prime \prime \prime} \approx_{2} H^{\prime \prime}$, and we get:

$H^{\prime \prime \prime} \approx_{2} H^{\prime \prime} \approx_{2} H^{\prime} \approx_{1} H$, so $H^{\prime \prime \prime} \approx_{2} H^{\prime} \approx_{1} H$. Since $H^{\prime \prime \prime} \forall P_{1}$ then $H \not K_{1} K_{2} P_{1}$, hence $H \notin K_{1} K_{2} K_{1} P_{1}$.

It seems reasonable to suppose that a back and forth interchange of messages will only make a finite amount of difference and we proceed to show now that this is indeed the case.

Theorem 12. Let $A$ be a formula of the form $x B$, where $x$ is a string of knowledge operators and $B$ is a boolean combination of private facts $P_{i}$. Let $H \forall K_{j} A$. Then if $H^{\prime \prime}=H ; H^{\prime}$ and $H^{\prime \prime}=K_{j} A$, ihen there is a receive in $\Phi_{j}\left(H^{\prime}\right)($ either of the form of $r(j, l, m)$ for some $l$, or $b c(l, U, m)$ where $j \in U, l \neq j)$. Informally: a process may learn something about the others only when it receives a message. 
Proof. Proof by induction on the length of $H^{\prime}$. If the length of $H^{\prime}$ is 0 then the theorem clearly holds. So suppose that $H^{\prime}$ is $H_{0}^{\prime} ; G$. If $H ; H_{0}^{\prime} \not K_{j} A$ then there is some $H_{1} \approx{ }_{j} H ; H_{0}^{\prime}$ such that $H_{1} \not A$. By the splitting rule if $G$ is not a broadcast, we can split it: there are $G_{2}, G_{1}$ such that $H ; H_{0}^{\prime} ; G_{2} ; G_{1}$ is in P where $\left(G_{1}\right)_{j}=(G)_{j},\left(G_{1}\right)_{i}=$ null for $i \neq j ;\left(G_{2}\right)_{i}=(G)_{i}$, for $i \neq j,\left(G_{2}\right)_{j}=$ null. $H ; H_{0}^{\prime} ; G_{2} \approx_{j} H ; H_{0}^{\prime}$, so $H_{1} \approx_{j} H ; H_{0}^{\prime} ; G_{2}$. By the extension rule $H_{1} ; G_{1}$ must be in $\mathbf{P}\left(G_{1}\right.$ was not a receive!). Furthermore $H_{1} ; G_{1} \approx_{j} H ; H^{\prime}$. If $x$ was empty ( $A$ is a boolean combination of private facts), then if $H_{1} \not A$, then $H_{1} ; G_{1} \not \neq A$. If $x$ is $K_{s} y$, then by the induction hypothesis since $s$ has not received any message in $G_{1}$ he didn't learn anything, so if $H_{1} \forall A$, then $H_{1} ; G_{1} \forall A$. So in any case $H ; H^{\prime}=H^{\prime \prime} \forall K_{j} A$.

As a consequence we get the following theorem, which is essentially Chandy \& Misra's (1986) theorem 5.

Theorem 13. (Chandy \& Misra 1986) If for some histories $H, H^{\prime}$ such that $H$ is an initial segment of $H^{\prime}$ :

$$
H^{\prime} \vDash K_{i(1)} K_{i(2)} \cdots K_{i(p)} A \text { and } H \not K_{i(p)} A
$$

then in $H^{\prime}-H$ there must be a sequence of messages: $m_{p-1}, m_{p-2}, \ldots, m_{1}$ such that $m_{p-1}$ is sent by $i(p)$ and reaches $i(p-1)$ (maybe via some other processes), $\ldots, m_{i(1)}$ is sent by $i(2)$ and (maybe indirectly) reaches $i(1)$ (the messages may be different but they must all imply $A$ ). Moreover if $A$ doesn't depend on any local event of $i(p)$ (its truth value depends on some event $\left.e \notin E_{i(p)}\right)$ then there must be some event of the form $r(i(p), l, m)$ occurring after $H$ but before $s\left(i(p), i(p-1), m_{p-1}\right)$.

Proof. By induction on $p$ using the previous theorem.

Theorem 14. Suppose communication is asynchronous and $A$ is a persistent formula. Let $H$ and $H^{\prime}$ be global histories such that $H^{\prime}$ extends $H$. Then $L\left(A, H^{\prime}\right)$ includes $L(A, H)$ and there is a finite $X$ such that $L\left(A, H^{\prime}\right) \subseteq d c(X ; L(A, H))$.

Proof. The fact that the level increases with time follows from the fact that we deal with persistent formulae.

For the second part we use the theorem of Chandy \& Misra (1986).

Since there are only finitely many events $G$ between $H$ and $H^{\prime}$, there are only finitely many possible sequences of events as above and a finite set of strings $x$ for which the conditions of theorem 13 are satisfied. Let $X$ be the set of these strings, then $X$ satisfies the conditions of the theorem.

Now we characterize precisely how the level of knowledge grows when a message is received.

Theorem 15. Suppose that a history $H$ is exactly the following sequence of messages:

$$
\begin{aligned}
& s\left(i_{m}, i_{m-1}, R_{m}\right) ; \quad r\left(i_{m-1}, i_{m}, R_{m}\right) \\
& s\left(i_{m-1}, i_{m-2}, K_{i(m)} R_{m}\right) ; \quad r\left(i_{m-2}, i_{m-1}, K_{i(m)} R_{m}\right) \ldots \\
& s\left(i_{2}, i_{1}, K_{i(3)} \cdots K_{i(m)} R_{m}\right) ; \quad r\left(i_{1}, i_{2}, K_{i(3)} \cdots K_{i(m)} R_{m}\right)
\end{aligned}
$$


(For simplicity we have left out the null events of processors), where $R_{m}$ is initially (in the empty history) known only to $i_{m}$; then

$$
L\left(R_{m}, H\right)=d c\left\{K_{i(1)} K_{i(2)} \cdots K_{i(m)}\right\} .
$$

Proof. It is easy to notice that $d c\left\{K_{i(1)} K_{i(2)} \cdots K_{i(m)}\right\} \subseteq L\left(R_{m}, H\right)$. The other inclusion we prove by induction on the length of $H$. If it is 0 then $H$ is empty so $R_{m}$ is known only to $i_{m}$. So assume that the theorem is true for histories of length up to $m-1$.

Let us assume then that there is some $y$ such that $H \vDash y R_{m}$ and

$$
y \notin d c\left\{K_{i(1)} K_{i(2)} \cdots K_{i(m)}\right\} \text {. }
$$

So $y$ must be non-empty. Let then $y=K_{l} y^{\prime}$. If $l$ was not mentioned in the sequence of messages ( $l$ was not any of $\left\{i_{1}, \ldots, i_{m}\right\}$ - then $l$ would have known $R_{m}$ initially (it hasn't received any messages, see theorem 12 ) - which contradicts our assumption. So it must be that $l \in\left\{i_{1}, \ldots, i_{m}\right\}$. Then we can express $K_{i(1)} K_{i(2)} \cdots K_{i(m)}$ as $z K_{t} z^{\prime}$ where the $K_{l}$ picked is the leftmost occurrence of $K_{l}$ in $K_{i(1)} K_{i(2)} \cdots K_{i(m)}$. Let's define $H^{\prime}$ as the initial segment of $H$ up to the last receive by $l$. l doesn't learn anything in $H-H^{\prime}$ (he doesn't receive any messages), so if $H=K_{l} y^{\prime} R_{m}$ then $H^{\prime} \vDash K_{l} y^{\prime} R_{m}, H^{\prime}=H^{\prime \prime} ; G$ where $(G)_{i}=r(l, s, m)$. By the splitting rule we can split $G$ into $G_{1}$ and $G_{2}$, where $G_{1}$ is a receive on $l, G_{2}$ is null on $l . H^{\prime \prime} ; G_{1} ; G_{2}$ must be in the protocol. $H^{\prime} \approx_{l} H^{\prime \prime} ; G_{1}$, so $H^{\prime \prime} \vDash y^{\prime} R_{m}$. Then by the induction hypothesis $y^{\prime} \in d c\left(\left\{z^{\prime}\right\}\right)$, so $y^{\prime} \leqslant z^{\prime}$ and therefore $y \preccurlyeq K_{i(1)} K_{i(2)} \cdots K_{i(m)}$.

Theorem 16. (a) Suppose $H$ realizes $L(A, H)$ (for some formula $A$ which is a boolean combination of $P_{i}$ ) and $H \leqslant H^{\prime 6}$, i.e. all messages in $H$ occur in $H^{\prime}$ and in the same order, then $H^{\prime}$ realizes at least $L(A, H)$.

(b) Suppose $H$ realizes $L(A, H)$ and $H^{\prime}$ realizes $L\left(A, H^{\prime}\right)$ Suppose that $H^{\prime \prime}$ is the concatenation of $H$ and $H^{\prime}$. Then:

$$
L\left(A, H^{\prime \prime}\right)=L(A, H) \cup L\left(A, H^{\prime}\right)
$$

Proof. Part (a) follows easily by induction on the length of $H$ using the fact that $A$ is persistent.

To see part (b), clearly $L(A, H) \cup L\left(A, H^{\prime}\right) \subseteq L\left(A, H^{\prime \prime}\right)$.

Suppose $H^{\prime \prime} \vDash y A$. If $y$ is empty, then we are done, since $H \vDash A$.

Otherwise let $y A$ be true in $H^{\prime \prime}$, where $y$ is (say) $K_{1} y^{\prime}$. Suppose that $K_{1} y^{\prime} \notin L(A, H)$. Then there must be some point $T$ such that $H ; H_{T} \vDash K_{1} y^{\prime} A, H ; H_{T^{\prime}} \forall K_{1} y^{\prime} A$ (for all $H_{T^{\prime}}$ such that $H_{T^{\prime}} \leqslant H_{T} \leqslant H^{\prime}$ ). By theorem $12 H_{T}$ has as its last event some $G$ such that $(G)_{1}=r(1, l, z A)$. So $y^{\prime} \preccurlyeq K_{l} z$. But the event $G$ was a part of $H^{\prime}$. Message sent and received in $H^{\prime}$ must have been true in $H^{\prime}$. So $H_{T} \vDash K_{1} y^{\prime} A$, so $H^{\prime} \vDash y A$.

Note that $H$ and $H^{\prime}$ could be executed in parallel. In fact we can take any minimal $H^{\prime \prime}$ such that both $H$ and $H^{\prime}$ are embeddable in $H^{\prime \prime}$.

We now show that all finite downward closed sets are actually attainable as knowledge levels $L(A)$ of formulas in asynchronous systems.

Theorem 17. Every finite downward closed set is the set $L(A, H)$ for an appropriate $A$ and $H$ in some asynchronous protocol.

\footnotetext{
${ }^{6} \leqslant$ is here embeddability relation defined for histories.
} 
Construction. Let $X$ be a finite downward closed set of strings from $\Sigma^{s}$. We construct a formula $A$ and a history $H$ such that $L(A, H)=X$.

(1) If $X$ is empty then $A$ is the formula false.

(2) If $X$ consists of the empty string, then $A$ is the conjunction $P_{1}$ and $P_{2}$ where: $P_{1}$ is a predicate whose truth value is initially known only to process $1, P_{2}$ is a predicate whose truth value is initially known only to process 2 , and $H$ is the empty history.

(3) Otherwise $X$ has nonempty strings and therefore the set of all maximal strings $M$ in $X$ is nonempty. Let $M=x_{1}, x_{2}, \ldots, x_{r}$. Let $x_{i}=K_{i\left(k_{i}\right)} \cdots K_{i(2)} K_{i(1)}$ for $i=1, \ldots, r$. Then we take $A$ to be a $\vee_{i=1}^{r} P_{i(1)}$.

Let

$$
\begin{gathered}
H_{i}=s(i(1), i(2), A) ; r(i(2), i(1), A) ; s\left(i(2), i(3), K_{i(1)} A\right) \\
\cdots s\left(i(k-1), i(k), K_{i(k-2)} \cdots K_{i(2)} K_{i(1)} A\right) \\
\\
r\left(i(k), i(k-1), K_{i(k-2)} \cdots K_{i(2)} K_{i(1)} A\right) .
\end{gathered}
$$

Clearly $L\left(A, H_{i}\right)=d c\left(x_{i}\right)$. Now by theorem 16 if $H=H_{1} ; H_{2} ; \ldots H_{r}$ then $L(A, H)=$ $\cup_{i=1}^{r} d c\left(x_{i}\right)$.

Note that $H$ could be any permutation of $H_{i}$ 's. In fact all $H_{i}$ could be executed in parallel.

\subsection{Synchronous case}

In this section we will augment the alphabet $\Sigma$ to a larger alphabet $\Sigma_{C}$ which includes symbols $C_{U}$ where $U \subseteq N$. Semantically, the symbol $C_{U}$ denotes the set $\left\{K_{i} \mid i \in U\right\}^{s}$ and will be referred to as the common knowledge of processors in $U$. However, the element $C_{U}$ of $\Sigma_{C}$ is not the infinite set of all strings in the denotation of $C_{U}$, but the string $C_{U}$ where $U$ is explicitly enumerated. The $C_{U}$ as part of a message is a finite string which denotes an infinite regular set of strings.

Levels of knowledge will continue to be identified with subsets of $\Sigma^{s}$. Thus, for example, the string $C_{1,2} C_{2,3}$ denotes, as a subset of $\Sigma^{s}$, the set of all simple strings consisting of any number of $K_{1}$ and $K_{2}$ followed by any number of $K_{2}$ and $K_{3}$.

We start with a preliminary result.

Theorem 18. The set $L(A)$ is infinite iff it includes common knowledge of $A$ between two distinct processes $i$ and $j$.

Proof. One direction is clear, as common knowledge between $i$ and $j$ includes all strings in $\left\{K_{i}, K_{j}\right\}^{s}$.

Conversely, suppose that $L(A)$ does not include common knowledge of any two distinct $i, j$. Then, since $L(A)$ is downward closed, for any such $i, j$, there must be a maximum $A l t(i, j)$ number of alternations between $K_{i}$ and $K_{j}$ in any string in $L(A)$. Let $m_{A}$ be the largest of these $A l t(i, j)$ and let $p$ be $m_{A} * n^{2}$ ( $n$ is the number of processors). Now any nonrepeating string of length greater than $p+1$ has at least $p+1$ alternations, and hence more than $m_{A}$ for some specific alternation between some $K_{i}$ and $K_{j}$. Thus no string in $L(A)$ can have length greater then $p+1$ and $L(A)$ is finite.

In the following theorem we recall that $C_{U}$ stands semantically for the set $\left\{K_{i} \mid i \in U\right\}^{s}$. 
Theorem 19. If we broadcast " $x A$ " where $x$ is a string of knowledge operators and $A$ is a boolean combination of propositions $P_{i}$ among the group of processes $U$, then the created level of knowledge of $A$ increases by the downward closure of $\left\{C_{U}\right\}$. Formally: If $H=H^{\prime} ; G, \forall j \in U(G)_{j}=b c(i, U, x A), \forall j^{\prime} \notin U(G)_{j^{\prime}}=$ null, then:

$$
L(A, H)=L\left(A, H^{\prime}\right) \cup d c\left(\left\{C_{U}\right\}\right) d c(\{x\})
$$

Proof. Let $H$ be as in the premises of this theorem. Then $H \vDash A$ (our processes are honest and $A$ may be broadcasted). Moreover since it is a property of all histories in the protocol, it is common knowledge that formulae sent (or broadcasted) are true and remain true (all formulae persistent):

$$
\vDash C_{N}\left(\left(H=H^{\prime} ; G ; H^{\prime \prime} \text { and }(G)_{j}=b c(i, U, x A)\right) \rightarrow H \vDash x A\right),
$$

this implies that for every $U \subseteq N$ :

$$
\vDash C_{U}\left(\left(H=H^{\prime} ; G ; H^{\prime \prime} \text { and }(G)_{j}=b c(i, U, x A)\right) \rightarrow H \vDash x A\right),
$$

so in order to prove that $H \vDash C_{U} x A$ it is enough (since common knowledge is closed under modus ponens) to show that $H \vDash C_{U}\left(H=H^{\prime} ; G ; H^{\prime \prime}\right)$ and $(G)_{j}=b c(i, U, x A)$. But it is a property of $\mathbf{P}$ that if for some $j \in U,(G)_{j}=b c(i, U, B)$ then for all $j \in U,(G)_{j}=$ $b c(i, U, B)$. So $\forall H_{0} \approx_{U^{*}} H\left(H=H^{\prime} ; G ; H^{\prime \prime}\right.$ and $\left.(G)_{j}=b c(i, U, x A)\right) \rightarrow\left(H_{0}=H_{0}^{\prime} ; G ; H_{0}^{\prime \prime}\right.$ and $\left.(G)_{j}=b c(i, U, x A)\right)$. Therefore $H \vDash C_{U} x A$ so $H \vDash d c\left(\left\{C_{U}\right\}\right) d c(\{x\}) A, H \vDash d c\left(\left\{C_{U} x\right\}\right) A$. The theorem follows since no process outside of $U$ received any message.

We now prove that in the presence of synchronous communication, if there are at least three processors, then every downward closed set of strings without repetitions is the level of knowledge of some formula under some history. We have proved that if $m=\left(x_{1}, \ldots \lambda, x_{k}\right)$ is the set of minimal elements of the complement of some downward closed set $L$, then

$$
\begin{aligned}
& L=\cap N\left(x_{i}\right): i \leqslant k . \\
& \text { If } x=a_{1} \cdots a_{m} \text { then } N(x)=\left(\Sigma-a_{1}\right)^{s} \cdots\left(\Sigma-a_{m}\right)^{s} .
\end{aligned}
$$

The next theorem will show us how to realize $N(x)$. We take a sequence of broadcasts to $N-1$ processes at a time. Let's assume that at time $T$, group $U_{T}$ receives a broadcast and at time $T+1$ group $U_{T+1}$ (both of $N-1$ processors). Then the processor sending broadcast at $T+1\left(a_{T+1}\right)$ will be one of the processors in $U_{T+1} \cap U_{T}$, and the message sent will be the string of common knowledge operators $C_{U_{T}} \cdots C_{U_{1}}$ followed by a fixed formula $P$ private to one of the processes in $\left(\Sigma-a_{m}\right)$.

Theorem 20. Let $H$ be a history in which the private information of $s_{1}$ is $P_{s}$, and every event $G^{T}$ occurring in $H$ at time $T$ for $T=1, \ldots, l$, (where $l=$ length $(H)$ ) is of the form: $\left(G^{T}\right)_{i}=b c\left(a_{s_{r}}, N-\left\{a_{T}\right\}, m_{T}\right)$ for all $a_{s_{T}} \neq a_{T}, a_{s_{T}} \neq a_{T-1},\left(G^{T}\right)_{i}=$ null otherwise. Where $m_{1}=P_{s_{1}}, m_{T+1}=C_{N-\left\{a_{T}\right\}} m_{T}$. Then:

$$
L\left(P_{s_{1}}, H\right)=d c\left(C_{N-\left\{a_{3}\right\}} \cdots C_{N-\left\{a_{1}\right\}}\right)
$$

Proof. Induction on the length of $H$. Clearly if $l=1$ then $L\left(P_{s_{1}}, H\right)=C_{N-\left\{a_{1}\right\}}$.

Suppose that for histories up to the length $l-1$ theorem is true. Now we can use 
theorem 19: if $H=H^{\prime} ; G^{l}$ where $\left(G^{l}\right)_{i}=b c\left(a_{s_{l}}, N-\left\{a_{l}\right\}, m_{l}\right)$ then $L\left(P_{s_{1}}, H\right)=L\left(P_{s_{1}}, H^{\prime}\right) \cup$ $\operatorname{dc}\left(C_{N-\left\{a_{1}\right\}} C_{N-\left\{a_{1}\right\}} \cdots C_{N-\left\{a_{1}\right\}}\right)$.

Since by induction hypothesis (IH) $L\left(P_{s_{1}}, H^{\prime}\right)=d c\left(C_{N-\left\{a_{t-1}\right\}} \cdots C_{N-\left\{a_{1}\right\}}\right)$ then

$$
L\left(P_{s_{1}}, H\right)=d c\left(C_{N-\left\{a_{i}\right\}} \cdots C_{N-\left\{a_{i}\right\}}\right)
$$

\section{COROLLARY 1}

In a system with at least 3 processors, for every $x$ in $\Sigma^{s}$, there exists a history $H$ and a formula $A$ such that $L(A, H)$ is just the set of strings without repetitions in $N(x)$.

Theorem 21. Every downward closed set $L$ of strings without repetitions is $L(A, H)$ for suitable $A$ and $H$ in a synchronous system with at least 3 processors.

Proof. Let $\left\{x_{1}, \ldots, x_{k}\right\}$ be the set of minimal elements of the set of strings without repetitions which are not in $L$. Then $L$ is $N\left(x_{1}, \ldots, x_{k}\right)$.

If $k=0$ then all strings without repetitions are in $L$ and $L$ is just common knowledge, which can be achieved by taking the formula $A$ to be a tautology.

Otherwise for each $x_{j}$ we can find a $P_{j}$ and a history $H_{j}$ such that $L\left(P_{j}, H_{j}\right)$ is exactly $N\left(x_{j}\right)$. Now let $H$ be $H_{1} ; \ldots ; H_{k}$ and $A$ be the conjunction of the $P_{j}$. (We assume the $P_{j}$ are all independent so that if $i \neq j$ then $H_{i}$ conveys no information about $P_{j}$.) Then $y \in L(A, H)$ iff $H \vDash y A$ iff for all $j, H \vDash y P_{j}$ iff for all $j, H_{j} \vDash y P_{j}$ iff for all $j, y \in N\left(x_{j}\right)$ iff $y \in L$.

Theorem 22. In a two processor system with only synchronous communication available, no finite level containing strings of length $\geqslant 2$ can be achieved for any formula $A$.

Proof. Suppose that $H \vDash K_{1} K_{2} A$. If in the empty history 1 knows that 2 knows $A$, then $A$ must be true in all histories ( $A$ persistent) and therefore must be common knowledge. Otherwise 1 must have learned that $K_{2} A$ in $H$, so there must have been a communication between 1 and 2 to that effect, but there are only synchronous communications in the system, and these create common knowledge.

\subsection{Mixed case-both kinds of communication available}

If we have both kinds of communications in the system, we can directly realize every level. We show first how to realize a level in the normal form. Later we will also show how to realize the level given by the set of minimal strings of the complement. As we will see from the examples in a following section, if we have some specification of the level of knowledge to be achieved, which is incomplete (doesn't specify a unique level), then the two constructions will give us different levels of knowledge (maximal and minimal) satisfying requirements.

\section{Construction}

$$
L=\bigcup_{i=1}^{k} d c\left(X_{i}\right)
$$

where $X_{i}$ is star-linear. 
We take $A=\vee_{i=1}^{k} P_{s(i)}$ where $s(i) \in U_{j}$, such that $x_{i(1)}=C_{U_{j}}$. We create $H_{i}$ to realize the level of knowledge $d c\left(X_{i}\right)$ of a formula $P_{s(i)}$ for every $i$, and then we take $H$ to be the concatenation of all $H_{i}$ 's.

We can also construct a level specified by a set of minimal strings of the complement.

\section{Construction.}

$$
L=N\left(x_{1}, \ldots, x_{k}\right)
$$

where $x_{i} \in \Sigma^{s}$.

We take $A=\wedge_{i=1}^{k} P_{s(i)}$ where $s(i) \in U_{j}$, such that $x_{i}=y C_{U_{j}}$. We create $H_{i}$ to realize the level of knowledge $N\left(x_{i}\right)$ of a formula $P_{s(i)}$ for every $i$, then we take $H$ to be a concatenation of all $H_{i}$ 's.

Let $x_{i}=C_{U_{m}} \cdots C_{U_{1}}$.

(i) $s(i)$ initially sends a broadcast $P_{s(i)}$ to all the processes not in $U_{1}$.

(ii) Sender of the last broadcast (to $N-U_{j}$ ) sends asynchronously all that he knows about $P_{s(i)}$ to one of the processes not in $U_{j+1}$ (if there was no broadcast yet, $s(i)$ is a sender).

(iii) Recipient of the last asynchronous message broadcasts all that he knows about $P_{s(i)}$ to all processes in $N-U_{j+1}$.

\subsection{Limited n-casts}

If in our system we have only a limited broadcast capability, then not all levels of knowledge can be achieved. Precisely:

Theorem 23. If in a system of $N$ processes, all the broadcasts are to groups of $n$ processes $(n<N)$, then no $C_{U} A$ can be realized for any $U$ with $|U|>n$, for any formula $A$ which is not true in all the histories.

Proof. Broadcast $b c(i, U, m)$ creates common knowledge of $m$ among $U$. A sequence of broadcasts of up to $n$ processes (say to $\left.U_{1}, \ldots, U_{k}\right)$ will create $\operatorname{dc}\left(C_{U_{k}} C_{U_{k-1}} \cdots C_{1}\right)$ where all $U_{i}$ for $i=1, \ldots, k$ have at most $n$ elements. Let $U^{\prime}$ be a set of $n^{\prime}$ elements where $n^{\prime}>n$. Then $C_{U^{\prime}} \notin d c\left(C_{U_{k}} C_{U_{k-1}} \cdots C_{1}\right)$, so it is not realized by a sequence of $k$ $n$-casts.

Theorem 24. In the usual version of CSP with '2-casts', $C_{v}(A)$ with $|U|>2$ cannot be achieved for any $A$ which was not common knowledge to begin with.

\section{Examples}

Every level of knowledge can be generated in an appropriate system. Practically there may be two kinds of reasons we want to obtain some particular level of knowledge.

(1) We want to have enough knowledge in the system so we specify which strings $L$ we want to have included in a realized level $L(A, H)$.

(2) We want to prevent certain processes from knowing some facts about the others. In such a situation we would specify a set $L^{\prime}$ of the strings we don't want to include in $L(A, H)$. Let us consider now a pair $\left(L, L^{\prime}\right)$ as a knowledge specification for our system, and consider how we might realize it. 
1. If $L^{\prime} \cap d c(L)$ is not empty them $\left(L, L^{\prime}\right)$ cannot be realized for any formula.

For example if $L=\left\{K_{1} K_{2} K_{3}\right\}, L^{\prime}=\left\{K_{1}, K_{3}\right\}$ then there is no system which realizes $\left(L, L^{\prime}\right)$.

So a necessary condition for realizability of $\left(L, L^{\prime}\right)$ is:

$$
\forall m \in L \forall m^{\prime} \in L^{\prime} \neg\left(m^{\prime} \leqslant m\right) .
$$

Now we have two possibilities:

2. If $d c\left(\Sigma^{s}-L^{\prime}\right)=d c(L)$ then $\left(L, L^{\prime}\right)$ uniquely specifies the level, which can be realized for an appropriate formula in an appropriate system.

Let us look at an example. We have some fact $F$, and we want to create a history $H$, such that $H \vDash K_{A} K_{B} F, H=K_{B} K_{C} F, H \vDash K_{C} K_{A} F$ (these three facts already imply that $H \vDash K_{A} F, H \vDash K_{B} F, H \vDash K_{C} F$, but we also want $H \vDash \neg\left(K_{A} K_{C} F\right), H \vDash \neg\left(K_{B} K_{A} F\right)$, and $H \vDash \neg\left(K_{C} K_{B} F\right)$. So our specification is $\left(L, L^{\prime}\right)$, where $L=\left\{K_{A} K_{B}, K_{B} K_{C}, K_{C} K_{A}\right\}$, $L^{\prime}=\left\{K_{A} K_{C}, K_{B} K_{A}, K_{C} K_{B}\right\}$. Notice that in this case, since we exclude the possibility of common knowledge between any pair of processes, our level of knowledge must be finite. In fact these requirements characterize the level of knowledge completely. The level we are looking for is exactly the set $L(A, H)=\left\{K_{A} K_{B}, K_{B} K_{C}, K_{C} K_{A}, K_{A}, K_{B}, K_{C}, \varepsilon\right\}$.

We can attain ( $\left.L, L^{\prime}\right)$ by a protocol in which (for example) all $A, B, C$ independently learn about $F, A$ sends a message $F$ to $C, C$ sends $F$ to $B, B$ sends $F$ to $A$, and all messages are sent asynchronously. In a synchronous system we can achieve $\left(L, L^{\prime}\right)$ by a protocol in which first we use a broadcast to bring about common knowledge of the fact $(p \wedge q \wedge r) \rightarrow F$. $p$ is a private fact of $A, q$ is a private fact of $B, r$ is a private fact of $C$. A broadcasts $p$ to $\{A, B\}$ and later $B$ broadcasts $C_{\{A, B\}} p$ to $\{B, C\}$ creating $N\left(K_{A} K_{C}\right)$. Similarly first $B$ broadcasts $q$ to $\{B, C\}$, later $C$ broadcasts $C_{\{B, C\}} q$ to $\{A, C\}$ creating $N\left(K_{B} K_{A}\right)$, finally $C$ broadcasts $r$ to $\{A, C\}$, and then $A$ broadcasts $C_{\{A, C\}}{ }^{r}$ to $\{A, B\}$, creating $N\left(K_{C} K_{B}\right)$.

3. If $\left(L, L^{\prime}\right)$ can be realized but there is $x \in \Sigma^{s}$ such that $x \notin d c(L)$ and $\neg\left(\exists m^{\prime} \in L^{\prime}, m^{\prime} \leqslant x\right)$ then the level is not uniquely specified (we can include $x$ in $\left(L, L^{\prime}\right)$ but we don't have to).

The smallest level realizing $\left(L, L^{\prime}\right)$ is $d c(L)$ and the largest one is $N\left(L^{\prime}\right)$ (or $\left.d c\left(\Sigma^{s}-L^{\prime}\right)\right)$.

Let for example $L=\left\{C_{\{1,2\}} K_{3}\right\}, L^{\prime}=\left\{K_{3} K_{2}\right\}$. In order to realize $\left(L, L^{\prime}\right)$ we can take $d c(L)$. This can be done by taking formula $A$ to be $P_{3}$, and a history:

$$
s\left(3,1, K_{3} P_{3}\right) ; r\left(1,3, K_{3} P_{3}\right) ; b c\left(1,\{1,2\}, K_{3} P_{3}\right) \text {. }
$$

Another possibility is to realize $N\left(K_{3} K_{2}\right)$.

We thank Konstantinos Georgatos for a very careful reading of the manuscipt.

\section{References}

Aumann R 1976 Agreeing to disagree. Ann. Stat. 4: 1236-1239

Chandy M, Misra J 1986 How processes learn. Distrib. Comput. 1(1): 40-52

de Jongh D H J, Parikh R 1977 Well partial orderings and hierarchies. Proc. K. Ned. Akad. Wet. A80: 195-207

Halpern J, Moses Y 1990 Knowledge and common knowledge in a distributed environment. J. Assoc. Comput. Mach. 37: 549-578 
Halpern J, Zuck L 1987 A little knowledge goes a long way: simple knowledge-based derivations and correctness proofs for a family of protocols. Proc. 6th ACM Symp. on Principles of Distributed Computing (New York: ACM Press) pp. 269-280

Higman G 1952 Ordering by divisibility in abstract algebras. Proc. Lon. Math. Soc. 2: 326-336 Lamport L 1978 Time, clocks and the ordering of events in a distributed system. Commun. $A C M$ 21: 558-565

Lewis D 1969 Convention, a philosophical study (Harvard: University Press)

Moses Y, Tuttle M 1988 Programming simultaneous actions using common knowledge. Algorithmica 3: 121-169

Parikh R 1986 Levels of knowledge in distributed computing. IEEE Symposium on Logic in Computer Science (New York: IEEE Press) pp. 322-331

Parikh R, Krasucki P 1986 Levels of knowledge in distributed computing, Brooklyn College Dept. of CIS. Technical Report

Parikh R, Krasucki P 1990 Communication, consensus and knowledge. J. Econ. Theory 52: 178-189

Parikh R, Ramanujam R 1985 Distributed processes and the logic of knowledge. Logics of programs. Lecture Notes in Computer Science. Vol. 193 (Berlin: Springer-Verlag) pp. 256-268

Schiffer S 1972 Meaning (Oxford: University Press) 